\title{
Candida zeylanoides
}

National Cancer Institute

\section{Source}

National Cancer Institute. Candida zeylanoides. NCI Thesaurus. Code C114261.

A yeast in the phylum Ascomycota. This species is urease-negative, forms feather-like pseudohyphae consisting of chains of cells with oval blastospores, assimilates glucose, and does not grow in the presence of cycloheximide. C. zeylanoides is an opportunistic human pathogen which may cause fungemia and arthritis. 